\title{
Scaffold-free Scleraxis-programmed tendon progenitors aid in significantly enhanced repair of full-size Achilles tendon rupture
}

\begin{abstract}
Aim: Currently there is no effective approach to enhance tendon repair, hence we aimed to identify a suitable cell source for tendon engineering utilizing an established clinically relevant animal model for tendon injury. Materials \& methods: We compared, by in-depth histomorphometric evaluation, the regenerative potential of uncommitted human mesenchymal stem cells (hMSC) and Scleraxis (Scx)-programmed tendon progenitors (hMSC-Scx) in the healing of a full-size of rat Achilles tendon defect. Results: Our analyses clearly demonstrated that implantation of hMSC-Scx, in contrast to hMSC and empty defect, results in smaller diameters, negligible ectopic calcification and advanced cellular organization and matrix maturation in the injured tendons. Conclusion: Scaffold-free delivery of hMSC-Scx aids in enhanced repair in a clinically translatable Achilles tendon injury model.
\end{abstract}

First draft submitted: 20 October 2015; Accepted for publication: 16 February 2016; Published online: 13 April 2016

Keywords: mesenchymal stem cells $\bullet$ Scleraxis $\bullet$ tendon healing $\bullet$ tendon progenitors

Tendons injuries, consecutive to overuse or age-related degeneration, are common and present a clinical challenge in orthopedic medicine because of the lengthy periods of post-traumatic healing and functional gain, as well as the high rate of rupture recurrence $[1,2]$. Moreover, the number of tendon clinical interventions per year is constantly increasing, which is in direct relation to the rising population aging in Europe and the USA [3-5]. Certain tendons (Achilles, rotator cuff, patella and forearm extensors) are particularly susceptible to degeneration and surgical treatment. The standard therapeutic options for tendons repair comprise of suture, autografts, allografts and synthetic prostheses; however, none of these alternatives have provided a successful long-term solution, especially in aged individuals [6].

Tendon tissue heals by initial formation of poor scar tissue which is then gradually remodeled over long periods of time [7-12]. In short, the healing involves three overlapping phases: an inflammatory stage, which occurs immediately after the tendon rupture; a proliferation stage begins within the first days/ week and results in the of establishment scar tissue reconnecting the injured tendon ends; and a long remodeling stage including the two substages; consolidation and maturation, initiating around 6 weeks after injury and goes up to $1-2$ years depending on the age and condition of the patient [7-12]. Cellularity and matrix production are gradually decreased during the consolidation stage followed by gradual increase of collagen fibril alignment and cross-linking over the extended period of the maturation phase [712]. And precisely because tendon repair and rehabilitation requires lengthy periods, the field's great interest is to accelerate these processes without loss of quality and function the de novo built tissue. Cell-based tissue engineering is nowadays one of the most attractive and widely explored strategies for musculoskeletal regeneration. This approach
Chi-Fen Hsieh', Paolo Alberton', Eva LoffredoVerde ${ }^{1}$, Elias Volkmer ${ }^{1}$, Matthias Pietschmann², Peter Müller ${ }^{2}$, Matthias Schieker ${ }^{1}$ \& Denitsa Docheva*,1

'Experimental Surgery \& Regenerative Medicine, Department of Surgery, Ludwig-Maximilians-University (LMU), Nussbaumstr. 20, 80336 Munich, Germany

${ }^{2}$ Department of Orthopaedic Surgery, Physical Medicine \& Rehabilitation, University Hospital Grosshadern, LMU, Marchioninistr. 15, 81377 Munich, Germany

*Author for correspondence:

Tel.: +49 89/440055486

Fax: +49 89/440055489

denitsa.docheva@med.uni-muenchen.de

$\begin{array}{ll}\text { Future } & \mathrm{fS} \\ \text { Medicine } & \end{array}$ 
(A)

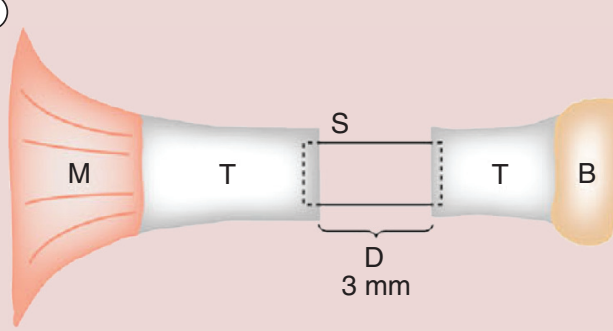

(B)

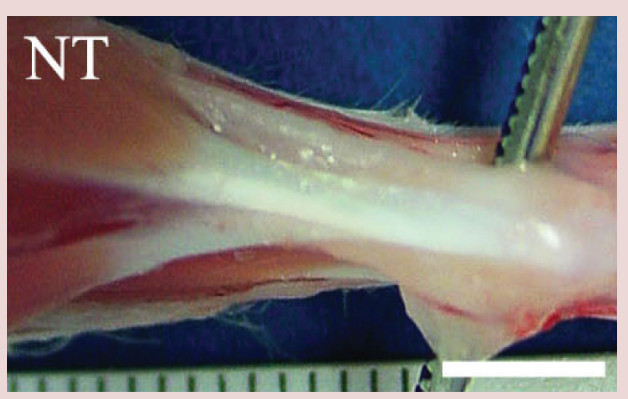

\section{hiMSC-Mock}

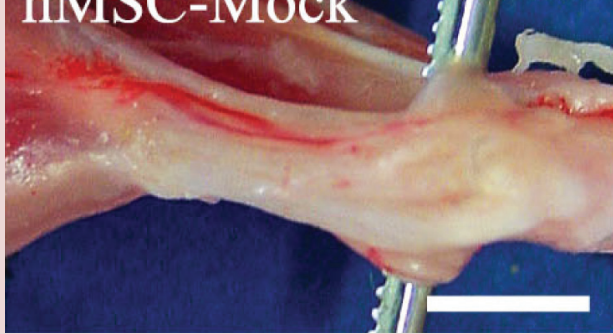

(C)

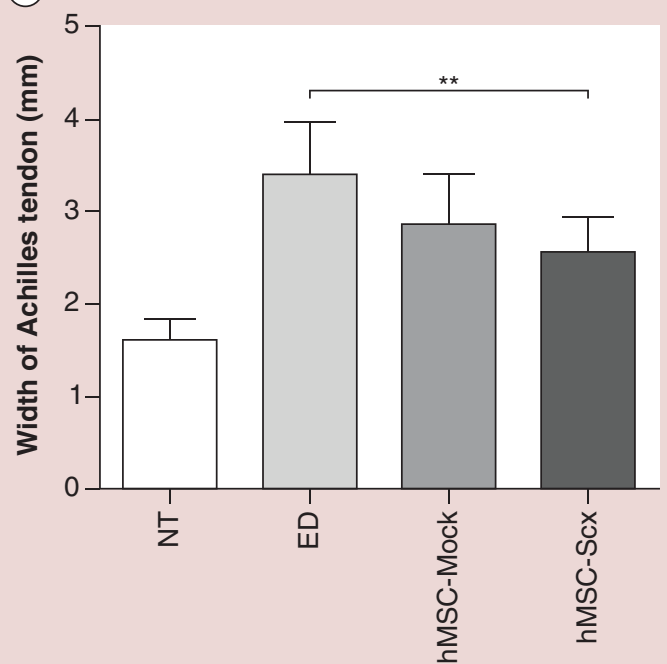

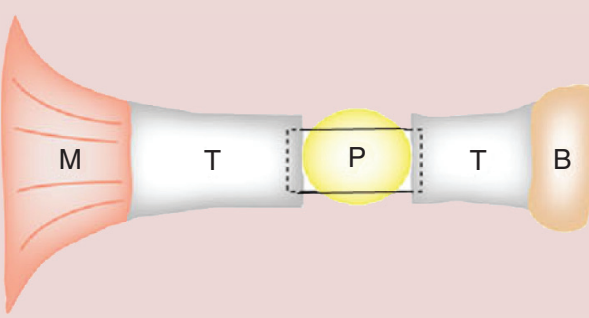
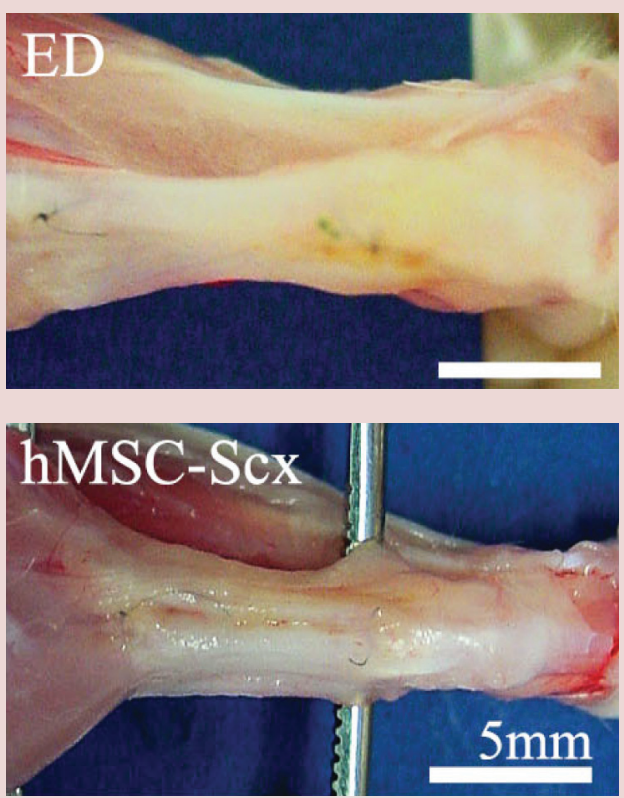

(D)

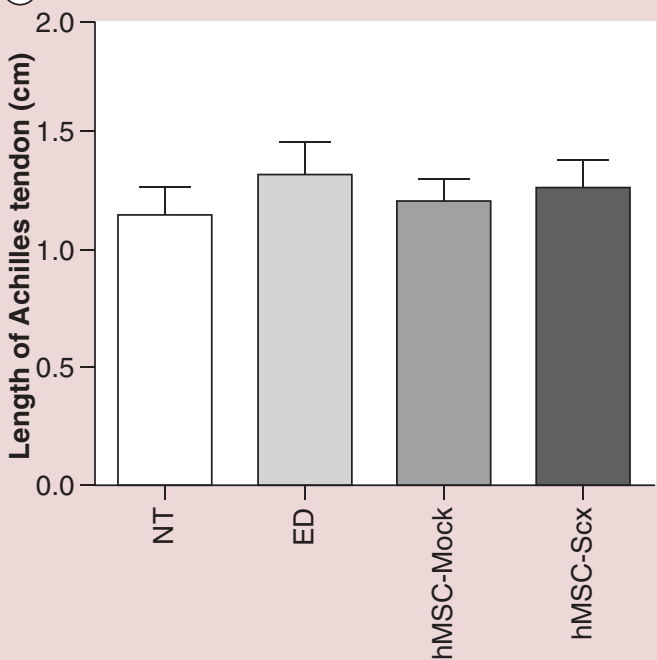

Figure 1. Investigation of Achilles tendon overall appearance and dimensions. (A) A schematic image of the established Achilles tendon injury model. (B) Representative gross morphological images of the four study groups, NT, ED, hMSC-Mock and hMSC-Scx after 16 weeks of healing. (C) The width, measured in the tendon proper, and (D) length of the Achilles tendons from the four groups. Bar chart present mean values \pm standard deviations. $* * p=0.002$ hMSC-Scx vs ED ( $n=10,10$ animals/group).

B: Calcaneus bone; D: Surgery defect; M: Gastrocnemius muscle; P: Cell pellet; S: Surgical suture; T: Achilles tendon. 
relies on reparative cells, alone or in combination with biocompatible scaffolds, which are delivered during patient's surgery into the site of tissue damage. Therefore, selecting the most appropriate cell type is of utmost importance.

In tendon engineering, several cell types such as mesenchymal stem cells (MSC) from different tissue sources and tendon-derived cells have been suggested as suitable targets. However, the involvement of each of these cell types has to solve various challenges (reviewed in $[7]$ ). Bone marrow (BM)-MSC are the most frequently evaluated cell type due to well-established collection protocols as well as being the best characterized cells; however, ectopic bone formation within the injured tendons is closely associated with BM-MSC implantation (reviewed in [7]). The main risks of autologous tendon cell applications are patient comorbidity and a phenotypic drift of the primary cells during the steps of in vitro expansion and validation $([13,14]$, and reviewed in $[7])$.

Scleraxis $(S c x)$ is a member of the basic helix-loophelix (bHLH) transcription factor family, which regulates embryonic tendon formation and postnatal tendon function [15,16]. In 2012, we showed that a stable ectopic expression of Scx in BM-hMSC resulted in the generation of a homogeneous population of committed tendon progenitors [17]. In 2013 [18], we published the establishment of a very clinically relevant fullsize Achilles tendon injury model in Rattus norvegicus. Recently, three studies, namely by Gulotta et al., Chen $e t a l$. and Tan $e t$ al., have tested whether $S c x$ in combination with cells and carriers can promote early tendon repair up in animal models [19-21]. Hence, in our study we aimed to fully explore the potential of a scaffold-free delivery of hMSC-Scx cells in contributing to tendon repair by utilizing the above in vivo model [18] and carrying out in-depth histo-morphometric examinations after 16 weeks of cell implantation. We selected this time point in order to assess the long-term effect of the cells and how advanced the critical late remodeling phase of tendon healing is.

\section{Materials \& methods \\ Cell culture}

The well-established and characterized hMSC-Mock and hMSC-Scx cell lines were used in the study (described in [17]). These two cell lines were maintained in Alpha minimum essential medium GlutaMAX culture media (Gibco, Germany) supplemented with $10 \%$ fetal bovine serum (FBS, Sigma-Aldrich, Germany). Cells were cultured on polystyrene dishes in a humidified incubator at $5 \% \mathrm{CO}_{2}$ and $37^{\circ} \mathrm{C}$. Cells were expanded to sufficient for implantation cell numbers in three-five consecutive passages. Prior implantation, aliquots of hMSC-Mock and hMSCScx were used for RNA isolation and polymerase chain reaction (PCR) validation of transgene expression (Supplementary Figure 1 \& Supplementary data), which has been previously demonstrated in [17].

\section{Animal model \& surgical procedure}

Surgical procedures were performed according to our established in vivo model with slight modifications [18]. All procedures for animal handling prior, during and after surgery were approved by the Animal Care and Use Committee of the Bavarian Government (animal grant no. 55.2-1-54-2531-58-08). Briefly, athymic female Foxn1nu rats were purchased from Charles River (Sulzfeld, Germany) and accommodated in the central animal facility. Four different study groups with ten rats per group were randomly selected from the colony: (1) Native tendon (NT), consisting of noninjured left Achilles tendon; (2) Empty defect (ED) group, consisting of injured right Achilles tendon without cell implantation; (3) and (4) in which the animals received one-shot implantation of scaffoldfree Mock- (hMSC-Mock group) or Scx-hMSC pellets (hMSC-Scx group) within injured right Achilles tendon, respectively. Cell centrifugation at $500 \mathrm{~g}$ for 10 min was carried out to form a $3 \mathrm{D}$ cell pellet comprising of $1 \times 10^{6}$ cells. The surgical operation consisted of surgically removing three millimeters tendon tissue from the Achilles tendon proper $(\sim 3 \mathrm{~mm}$ proximal from the calcaneus and $3 \mathrm{~mm}$ distal from the gastrocnemius muscle, average length of rat Achilles tendon is $12 \mathrm{~mm}$ ) (Figure 1A). Each defect was first reconnected by Kirchmayr-Kessler suture and then the 3D cell pellet was administrated with a cut sterile pipette tip and left to adhere for approximately $5 \mathrm{~min}$ (Figure 1A).

At 16 weeks after surgery animals were euthanased, gastrocnemius muscle - Achilles tendon - calcaneus bone units revealed by skin incision, imaged and width of tendon proper and total length of the Achilles tendons were manually measured. Afterward tissue samples were explanted and processed as described below.

\section{Histomorphometry}

The gastrocnemius muscle - Achilles tendon - calcaneus bone units were fixed either in $4 \%$ paraformaldehyde (PFA; Merck, Germany) or in $95 \%$ ethanol $/ 5 \%$ absolute acetic acid overnight at $4^{\circ} \mathrm{C}$ (each five animals/group). PFA-fixed sections were used for general histological and immunohistochemical stainings, while ethanol-fixed sections were used for immunohistochemistry with anticollagen antibodies (Supplementary Table 1). After fixation, specimens were decalcified in 10\% EDTA/PBS pH 8.0 (SigmaAldrich, Germany) for four weeks at room tempera- 
Point-based scoring system for histological and elastic fiber assessment in order to evaluate the progress of Achilles tendon repair.

\begin{tabular}{|c|c|c|c|c|c|c|c|c|}
\hline \multirow{4}{*}{$\begin{array}{l}\text { Study } \\
\text { group }\end{array}$} & \multicolumn{8}{|c|}{ Scored parameters } \\
\hline & \multirow{3}{*}{$\begin{array}{l}\text { I } \\
\text { Cellularity }\end{array}$} & \multirow{3}{*}{$\begin{array}{l}\text { II } \\
\text { Ectopic } \\
\text { calcification }\end{array}$} & \multirow{3}{*}{$\begin{array}{l}\text { III } \\
\text { Vascularized } \\
\text { areas }\end{array}$} & \multirow{3}{*}{$\begin{array}{l}\text { IV } \\
\text { Adipose } \\
\text { areas }\end{array}$} & \multirow{3}{*}{$\begin{array}{l}\text { V } \\
\text { Poor fibrous } \\
\text { tissue }\end{array}$} & \multirow{2}{*}{\multicolumn{2}{|c|}{$\begin{array}{l}\text { VI } \\
\text { Elastic fibers }\end{array}$}} & \multirow{3}{*}{$\begin{array}{l}\text { VII } \\
\text { Total points }\end{array}$} \\
\hline & & & & & & & & \\
\hline & & & & & & Content & Morphology & \\
\hline \multicolumn{9}{|l|}{ NT } \\
\hline Mean & 4 & 4 & 3.5 & 2.95 & 4 & 4 & 4 & 26.45 \\
\hline IQR & 0 & 0 & 1 & 1.3 & 0 & 0 & 0 & \\
\hline \multicolumn{9}{|l|}{ ED } \\
\hline Mean & 1 & 2.7 & 3.17 & 2.73 & 1.77 & 2.25 & 1.25 & 14.87 \\
\hline Median & 1 & 3 & 3 & 2.6 & 1.65 & 2.5 & 1 & 14.75 \\
\hline Median & 1.6 & 3 & 3 & 2.6 & 1.3 & 2.5 & 1.5 & 15.5 \\
\hline IQR & 1.3 & 1.3 & 1.2 & 0.65 & 1.1 & 1 & 1.75 & \\
\hline \multicolumn{9}{|c|}{ hMSC-Scx } \\
\hline Mean & 1.5 & 3.33 & 2.8 & 2.9 & 2.48 & 2.75 & 2.25 & 18.01 \\
\hline Median & 1 & 3.15 & 2.8 & 3 & 2.65 & 3 & 2.5 & 18.1 \\
\hline IQR & 1.5 & 0.825 & 1.6 & 0.3 & 2.78 & 0.75 & 1 & \\
\hline \multicolumn{9}{|c|}{$\begin{array}{l}\text { Mean, median point values and IQR (interquartile range) of scored points are shown. } \\
\text { I: Estimation of increase in cellularity compared with normal, uninjured tendon (NT). } \\
\text { II: Estimation of increase in areas with ectopic calcification compared with NT. } \\
\text { III: Estimation of increase in vascularized areas compared with NT. } \\
\text { IV: Estimation of increase in adipose-rich areas compared with NT. } \\
\text { V: Estimation of increase in areas with poor fiber organization compared with NT. } \\
\text { VI: Estimation of increase in total amount of mature elastic fibers compared with NT. } \\
\text { VII: The sum of points of classes I-VI. } \\
4 \text { points }=0-25 \%, 3 \text { points }=26-50 \%, 2 \text { points }=51-75 \% \text { and } 1 \text { point }=76-100 \% \text {. For elastin fiber morphology } 4 \text { points = thin parallel elastin fibers, while } 1 \text { point } \\
=\text { thick irregular elastin fibers. }\end{array}$} \\
\hline
\end{tabular}

ture, then treated with consecutive 10,15 and $20 \%$ sucrose/PBS solutions at $4^{\circ} \mathrm{C}$ for $2 \mathrm{~h}$ and last, embedded in tissue freezing medium (Jung, Germany), and cryosectioned longitudinally at $12 \mu \mathrm{m}$ (Microm HM500 OM, Germany) allowing full exposure of the whole complex Gastrocnemius muscle- Achilles tendon-calcaneus. Prior use sections were stored at $-20^{\circ} \mathrm{C}$.

Selected sections (PFA-fixed) were stained with hematoxylin and eosin (H\&E) [18], or periodic acidschiff (PAS) using well-established standard histological protocols or following manufacturer's instructions (PAS staining system, Sigma-Aldrich). For the Safranin-O/Fast Green (S.O/F.G) staining [18,22], the sections were equilibrated to room temperature and hydrated with PBS $3 \times 5$ min and then changed to deionized water. Next, the sections were placed in $0.02 \%$ F.G (Sigma-Aldrich) in 95\% ethanol for 12 min, rinsed under $1 \%$ Acetic Acid for $5 \mathrm{~min}$, immersed in $0.5 \%$ S.O (Sigma-Aldrich) for $15 \mathrm{~min}$. Last, samples were rinsed quickly in $90 \%$ and $100 \%$ ethanol, and mounted with Entellan mounting medium (Merck).

For immunohistochemistry, selected sections were treated with $2 \mathrm{mg} / \mathrm{ml}$ hyaluronidase (Sigma-Aldrich, Germany) for $30 \mathrm{~min}$ at $37^{\circ} \mathrm{C}$. After washing and blocking, primary antibodies were applied overnight at $4^{\circ} \mathrm{C}$. Next day, corresponding secondary antibodies were used for $1 \mathrm{~h}$. For full details on blocking solutions and antibodies used in the study refer to Supplementary Table 1. For immunofluorescencebased experiments, sections were shortly counterstained with 4',6-diamidino-2-phenylindole (DAPI) (Life technology, USA) and mounted with fluoroshield (Sigma-Aldrich, Germany). For 3,3' diaminobenzidine (DAB)-based experiments, an initial quenching step was performed using $4 \% \mathrm{H}_{2} \mathrm{O}_{2}$ /methanol for 30 $\mathrm{min}$ at room temperature, and afterward the classical 
vectastain $A B C$ kit was implemented according to the manufacturer's instructions (VectorLab, USA). Photomicrographs were taken with a black/white Axiocam $\mathrm{MRm}$ or color AxiocamICc3 camera mounted on Observer Z1 microscope (Carl Zeiss, Germany). Individual staining types were reproduced three-times independently and for each studied parameter according to the initial fixation, three to five animals per group were analyzed.

In order to evaluate the progress of tendon repair, H\&E- or consecutive elastin-stained sections from five animals (each animal represented with three to five different slides consisting of three to four sections) were employed for microscopical scoring by four independent observers in a blinded fashion. A point-based scoring system for histological and elastic fiber assessment was used similar to Stoll et al. [23] with some modifications. The following parameters were scored and given points: (I) percentage of increase in cellularity compared with NT; (II) percentage of increase in ectopic calcification; (III) percentage of vascularized areas versus total area; (IV) percentage of adipose-likeoccupied areas; (V) percentage of poor fibrous areas; (VI) elastin fiber content (percentage increase) and morphology; and (VII) total score as the sum of points in parameters I-VI (Table 1).

\section{Quantification of nuclear aspect ratio, orientation \& density}

In healthy tendon or in advanced stage of tendon healing, low numbers of cells with much flattened nuclei that are parallel-aligned to the tensile axis are typical features. To determine the aspect ratio of nuclei (NAR, defined as the ratio of the minor diameter to the maximal diameter) and cell density via nuclear count, DAPI images were analyzed using ImageJ software v1.45s with installed NII plugin (National Institutes of Health, MD, USA). First, by color threshold adjustment the images were converted in black and white wherein DAPI-stained areas appear in black color and the remaining background in white color. Second, by implementing 'analyze particles' tool in NII plugin nuclear numbers and minor and maximal diameter for each nucleus were automatically calculated. Third, NAR were next calculated with GraphPad software and distributed by frequency as flattened cells are represented by NAR $=0$, while rounded cells have NAR $=1$. Cell density was expressed by calculating the number of nuclei per $\mathrm{mm}^{2}$.

To determine nuclear orientation (by estimating angular deviation), H\&E-stained images were analyzed with the following algorithm using the AxioVision V4.8 software (Carl Zeiss): first, each image was converted to grey scale and nuclei were marked by adjusting the intensity segmentation threshold; second, nuclear orientation measurements were made with the 'angular' tool as the angle between the major axis of a nucleus and the longitudinal axis of the tissue were automatically given for manually selected nuclei. Nuclear orientation angle values of $0^{\circ}$ represent a nucleus that is perfectly aligned along the longitudinal axis and as this value increases toward $90^{\circ}$, the nucleus becomes more angled to the long axis of the tendon.

Six to nine randomly taken $20 \times$ magnification images per section (within the area of injury $3 \mathrm{~mm}$ proximal from the calcaneus) of three to five animals per group were analyzed and a total number of approximately 2000 nuclei were measured for NAR and density, while approximately 200 nuclei per group were evaluated for angular deviation.

\section{X-ray analysis}

Dissected hind limbs from the four study groups (ten animals/group) were screened for in-tendinous calcification using $\mathrm{x}$-ray imaging with a Cabinet $\mathrm{x}$-ray Faxitron (Hewlett Packard, USA). Images were taken with $32 \mathrm{kV}, 2 \mathrm{~mA}$ and $2 \mathrm{~s}$ exposure time. White areas comparable with the calcaneus represented calcified areas. The total area of calcification in each animal was digitally quantified in each Achilles tendon using the 'polygonal' tool of Image Pro Plus software v4 (Media Cybernetics, USA). In addition, mean value and standard deviations were calculated for each study group.

\section{Polarized microscopic evaluation}

Organized collagen type I fibers have strong birefringence property when exposed to polarized light; hence, they can be visualized in orange to yellow colors using microscopy. All 10 animals per group were prescreened and four representative animals/group were selected for the best quality of the H\&E slides allowing for optimal and identical setting of the polarized microscopy imaging. Each animal was represented with one slide consisting of three sections. Microscopy was performed with $10 \times$ objective supplemented with a polarized filer mounted on Axioskope 2 microscope (Carl Zeiss). For optimal imaging the transmission axis of the analyzer was with an angle of $54^{\circ}$ to the axis of the polarizer in the analyzes of all samples. In order to cover the entire tendon territory approximately 50 consecutive images/section were taken manually in a mosaic manner and digitally stitched with Adobe Photoshop CS5 software (Adobe System, CA, USA). Next, total Achilles tendon area (denominator) was measured in pixel with 'magic wand' tool and by using 'color selecting' tool the yellow-to-orange-positive pixels (numerator), corresponding to highly organized collagen areas, were automatically quantified. Final data were expressed 
as a percentage of collagen birefringence and for each study group mean values and standard deviations were calculated.

\section{Detection of the implanted human cells in rat} Achilles tendons

For detection of the implanted hMSC-Mock or hMSC-Scx, tissue sections were stained with humanspecific (HLA)-ABC antibody as described above and in Supplementary Table 1. For the detection of human DNA in rat tendons genomic PCR-based method was used as follows: the material of five tissue sections from 10 animals/group was scraped, collected and lyzed with $20 \mathrm{mg} / \mathrm{ml}$ proteinase Kin PCR buffer (Merck) overnight at $55^{\circ} \mathrm{C}$. For controlling sample loading PCR for rat glyceraldehyde-3-phosphate dehydrogenase (rGAPDH, primers F5'-GACATGCCGCCTGGAGAAAC-3' and R5'-AGCCCAGGATGCCCTTTAGT-3') was performed using the following PCR program: $95^{\circ} \mathrm{C} / 5$ min; $95^{\circ} \mathrm{C} / 30 \mathrm{~s} ; 56^{\circ} \mathrm{C} / 30 \mathrm{~s} ; 72^{\circ} \mathrm{C} / 60 \mathrm{~s} ; 72^{\circ} \mathrm{C} / 1 \mathrm{~min}$; as steps 2-4 38 cycles. PCR with human specific genomic primers for amelogenin X (AMELX, primers F5'-CAGCTTCCCAGTTTAAGCTCT-3' and R5'-TCTCCTATACCACTTAGTCACT-3' [24]) was performed with touchdown program: $94^{\circ} \mathrm{C} / 5$ min; $94^{\circ} \mathrm{C} / 30$ s; $57^{\circ} \mathrm{C} / 30$ s; $4.72^{\circ} \mathrm{C} / 30$ s; $94^{\circ} \mathrm{C} / 30$ s; $47^{\circ} \mathrm{C} / 30 \mathrm{~s} ; 72^{\circ} \mathrm{C} / 30 \mathrm{~s} ; 72^{\circ} \mathrm{C} / 5 \mathrm{~min}$ as steps $2-4$ 10 cycles and steps 5-7 38 cycles. MGResearch PCR instrument (BioRad, Germany) was used. The PCR products were separated on $2 \%$ agarose gels, stained with ethidium bromide and visualized on a gel imager (Vilber Lourmat, Germany).

\section{Data \& statistics analysis}

Quantitative data and statistical significance were analyzed with GraphPad Prism 5 software (GraphPad, CA, USA). In general, each group was segregated in two halves due to fixation procedures; then three to five animals per group were examined, but the total histology slide and section numbers were kept equal between groups. In width, length, scoring and x-ray analyses all ten animals were evaluated. Bar charts show mean values and standard deviations. Multigroup statistical testing was performed with one-way ANOVA followed by post hoc Dunnett's test and t-test, and p-values $<0.05$ were considered statistically significant.

\section{Results}

Engineered Achilles tendons with Scleraxisprogrammed tendon progenitors are superior in tissue appearance \& composition

The gross morphological appearances of the Achilles tendons of the four experimental groups were evaluated and documented post-dissection. We concluded that all tendon defect ends, including the ED group, were completely reconnected. Among the three injured groups, the healed Achilles tendons of the hMSC-Scx group had the best opalescent tissue appearance that resembled the native tissue (Figure 1B). According to our quantitative data, the hMSC-Scx group had significantly smaller mean tendon width compared with the ED group (Figure 1C), whereas no significant differences were found regarding the mean tendon length (Figure 1D).

For examination of the general tendon composition, organization as well as for overall assessment of the healing stage first, H\&E (Figure 2A) and elastin stainings and imaging of the four groups were performed. Afterward a qualitative evaluation of the degree of repair in all injured Achilles tendons was carried out by point-based scoring of the following parameters (Supplementary Figure 2): cellularity, ectopic calcification, vascularized areas, adipose areas, poor fibrous areas and elastic fiber content and morphology. The results of four independent blinded observers are given in Table 1. Our scoring data clearly showed that the hMSC-Scx group (total mean of 18.01 and median of 18.1) has the highest scores compared with the other injured groups (ED group: total mean of 14.87 and median of 14.75; hMSC-Mock group: total mean of 14.97 and median of 15.5), indicating a superior composition of the healed Achilles tendons that is closer to the noninjured, healthy tendon NT group.

Very flattened cell nuclei, parallel alignment of the cells along the longitudinal strain axis and low cellularity are characteristic features of healthy and fully repaired tendon tissue. To investigate these three aspects, first we quantified the frequency distribution of nuclei with different flatness factor. This was performed by estimating the nuclear aspect ratio (NAR) as NAR, lower than 0.1 corresponds to completely flattened/elongated nucleus of differentiated tenocyte-like cell. We found a tendency that the frequency of NAR lower than 0.1 in hMSC-Scx group was above $20 \%$ and thus higher than that of ED and hMSC-Mock groups (Figure 2B). Next, we studied and displayed the frequency of the nuclear deviations of approximately 200 nuclei/group in order to assess the cellular alignments within the Achilles tendons of the four study groups (Figure 2C). In a NT more than $70 \%$ of the nuclei are aligned in the range of $0-10^{\circ}$ angles to the longitudinal axis, another $25 \%$ of the nuclei are in the range of $10-20^{\circ}$ angles. Interestingly, only in the hMSC-Scx group about $80 \%$ of the nuclei were within the angular deviation range of $0-20^{\circ}$, while in the ED and hMSCMock groups more than $50 \%$ of the nuclei had angular deviation of $30-70^{\circ}$. This finding became even more apparent when we calculated the average and maxi- 
mum values of the nuclear orientation angles, which are shown in the circular sectors (Figure 2C). Only the hMSC-Scx group had average and maximum values very close to that of the control NT group. Last, we analyzed by automated nuclear counting the tendon cell density since at the time period of 16 weeks postsurgery low cell density indicates advanced remodeling phase and overall healing process (Figure 2D). The cell densities within the engineered area of ED, hMSCMock and hMSC-Scx groups were higher than the NT group, still the cellular density of hMSC-Scx Achilles tendons was significantly lower than the other two injured groups (Figure 2D).

\section{Scleraxis-programmed tendon progenitors reduce the ectopic bone formation}

Our study in 2013 [18] revealed in-tendinous calcification in the repaired Achilles tendons, which we discussed is due to altered biomechanics of the injured tissue. Hence, it was very critical in our new model to examine if the implantation of hMSC-Scx cell will result in lowering the ectopic calcification. Using $\mathrm{x}$-ray imaging and subsequent quantification, we can clearly demonstrate that indeed the hMSC-Scx group had the least degree of calcification in comparison to the $\mathrm{ED}$ and hMSC-Mock groups (Figure 3A \& B). To further verify this substantial result we also performed immunostaining for the classical bone-marker osteopontin, which confirmed that the hMSC-Scx group had very negligible and scars osteopontin-positive areas (Figure 3C).

Since bone formation is closely related to vascularization, we investigated for endothelial and macrophage-rich areas by immunohistochemistry against CD31 and CD68, respectively (Figure 3D \& E). In the $\mathrm{ED}$ and hMSC-Mock groups, vessels were observed in the large calcified areas and in the tendon periphery, while in the NT and hMSC-Scx groups vessels were rare and only at the tendon periphery and sheets. CD68-expressing cells were very seldomly observed within vessels in the ED, hMSC-Mock and hMSCScx groups without any detectible difference, suggesting their involvement in the gradual vessel obliteration rather than in inflammation.

\section{Scleraxis-programmed tendon progenitors aid in enhanced tendon tissue maturation}

Increase of collagen I to collagen III ratio, development of very organized collagen fibers with strong birefringence and enrichment of aligned and denser elastin fibers are hallmarks of advanced remodeling and late maturation phase of the tendon repair process [7]. This is paralleled with interchange of certain proteoglycans in the tendon matrix, namely reduction in biglycan (regulator of early and thin collagen fibril formation) and upregulation of decorin and lumican levels (regulators of late and thick collagen fibril formation) as well as a general drop in the deposition of mucopolysaccharides and nontendinous glycosaminoglycans/ proteoglycans $[25,26]$. Hence, to study whether implantation of hMSC-Scx cells leads to faster tendon healing in terms of quicker entry into the maturation repair phase of the remodeling stage, we first analyzed the overall content of mucopolysaccharides and cartilagerelated glycosaminoglycans in the Achilles tendons of all groups by PAS and S.O./F.G. staining, respectively (Figure 4A \& B). Among the injured groups, the ED group showed very strong positive PAS staining as well as several S.O/F.G-rich areas suggesting that the grafting of cells gave an advantage in the tendon healing of the corresponding study groups. This inclination was further confirmed by polarized light microscopy analyses which demonstrated increased collagen birefringence areas, equivalent of more mature collagen fibers, in the Achilles tendons from the hMSCMock and hMSC-Scx groups compared with the ED (Figure 5A \& B). Last, detailed immunohistochemistry studies of six different antigens, precisely collagen type I and III, biglycan, decorin, lumican and elastin, revealed that their expression pattern in hMSC-Scx group resembled that of the NT group (Figure 6). This group had higher collagen I, decorin, lumican and elastin levels than the ED and hMSC-Mock groups. Very apparent difference was observed with regards to biglycan levels: ED group exhibited very high expression signals, while implantation of either hMSC-Mock or hMSC-Scx cells resulted in biglycan expression comparable to the levels in the NT group.

Altogether, based on our immunohistochemistry data, and together with the above-described results, we firmly concluded that implantation scaffold-free delivery of hMSC-Scx cells leads to advanced and superior healing in a very clinically relevant in vivo model of full-size Achilles tendon injury.

\section{The implanted human cells integrate $\&$ can survive 16 weeks in vivo}

To examine whether the implanted hMSC-Mock and hMSC-Scx cells were integrated in the Achilles tendons and a portion of them has survived during the given healing period of 16 weeks, we performed first human-specific PCR on genomic DNA isolated from cryosections of the three injured groups, wherein the ED group served as negative control. In Figure 7A it is shown that in 9 out of 10 animals we detected human DNA only in the study groups that were implanted with cells. Next, by performing immunohistochemistry against the human HLA-ABC epitope, we localized 
(A)
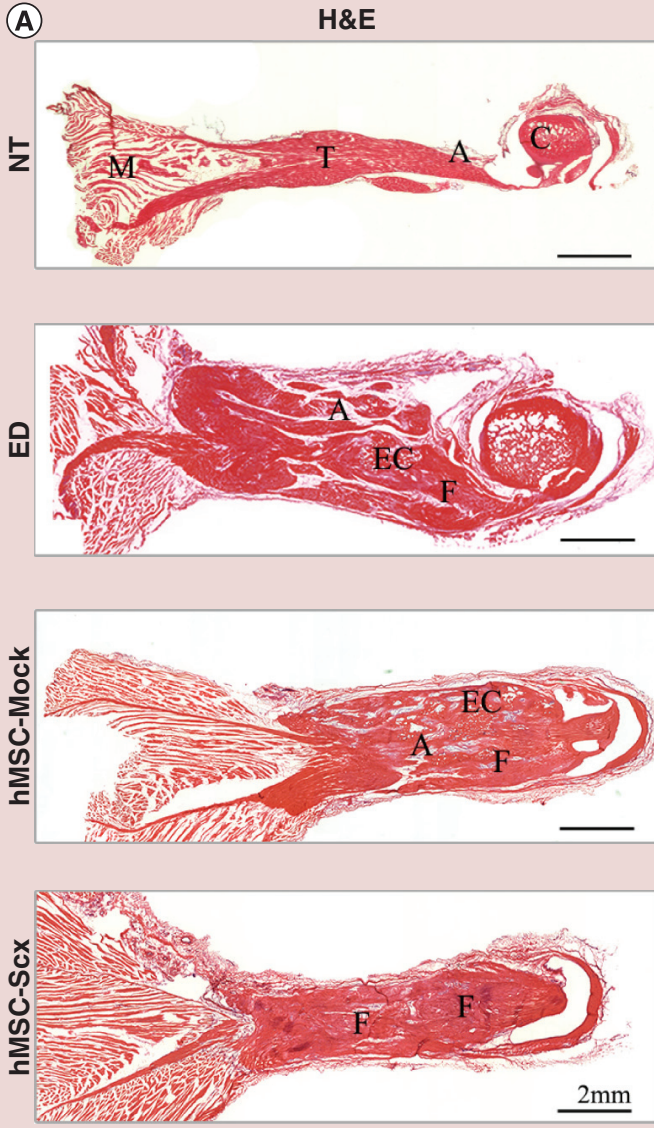

(D)

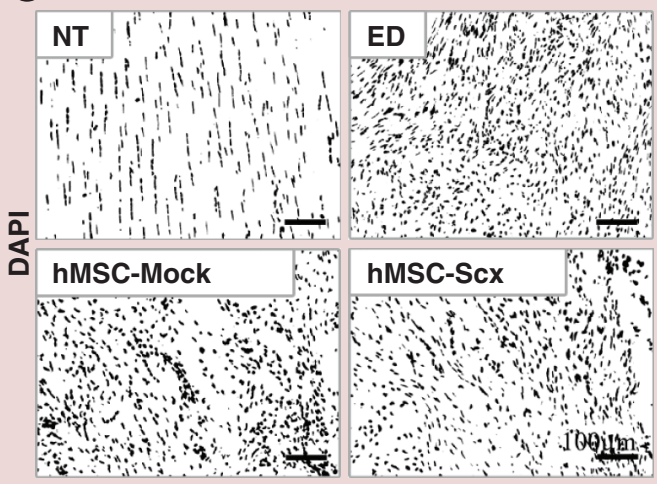

(B)
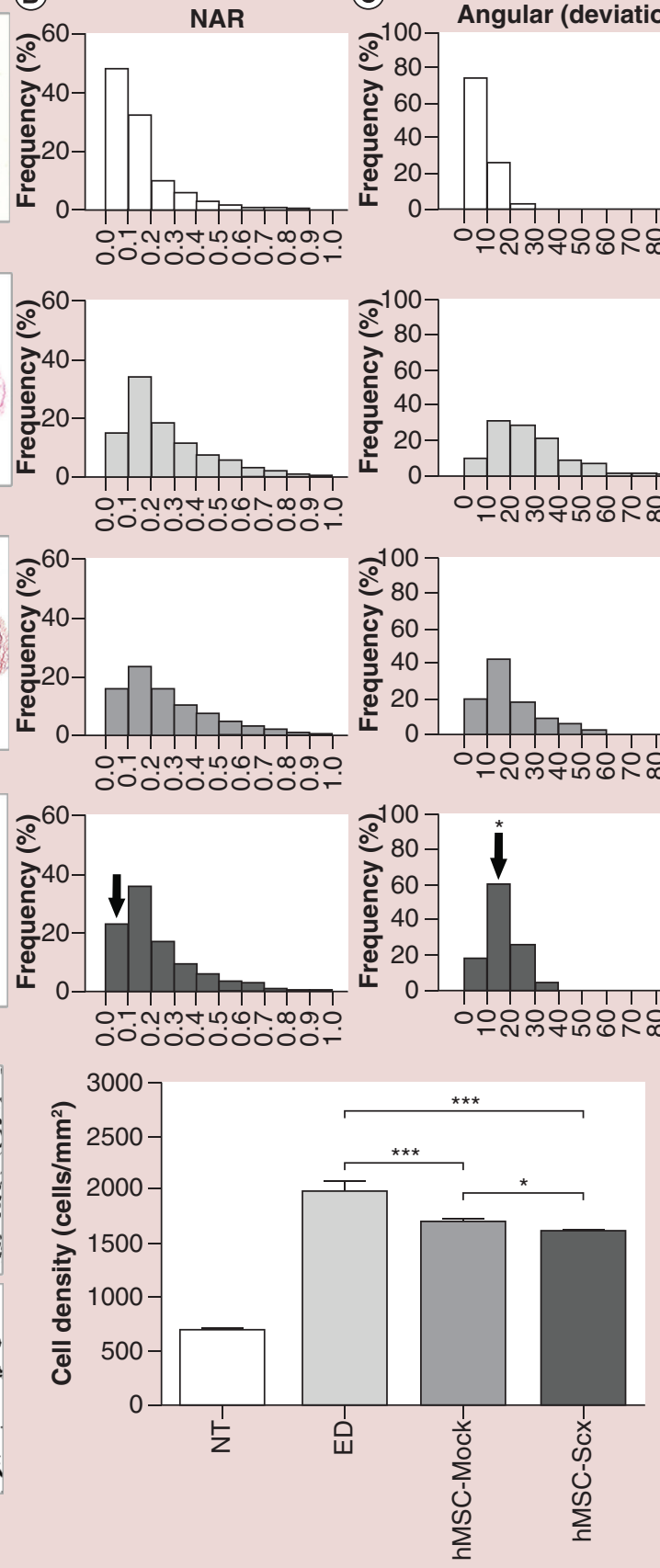

(C) Angular (deviation)
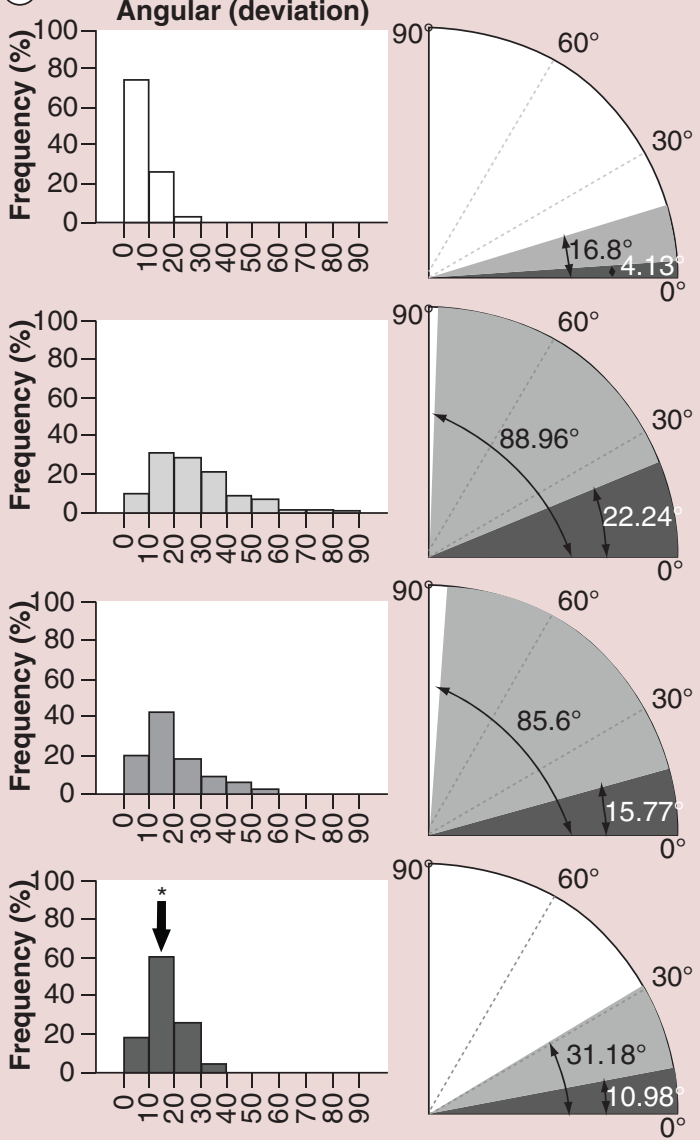

\begin{tabular}{|l|}
\hline Maximum \\
\hline Average value \\
\hline
\end{tabular}

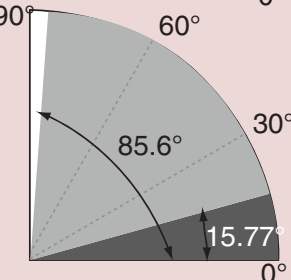

Figure 2. Detailed analyses of tendon tissue organization, nuclear aspect ratio, angular deviation and density. (A) Representative H\&E mosaic images for each study group displayed different substructures in the Achilles tendons, the extent of which were scored in Table 1. (B) Frequency distribution of nuclear aspect ratio (NAR) (width vs length of nucleus). The black arrow indicates a tendency in NAR 0.0-0.1 increase in hMSC-SCx versus ED. (C) Frequency distribution and circular sector of maximum and average value of angular deviation (angle between individual nuclear axis and tendon tissue longitudinal axis). The black arrow indicates * $p=0.05$ of hMSCScx versus ED in $10^{\circ}-20^{\circ}$ deviation. (D) Representative black and white-converted images of DAPI staining and chart of calculated cell density. Bar charts present mean \pm standard deviation; ***p $=0.0001$ hMSC-Scx versus ED, ***p $=0.0006$ hMSC-Mock versus ED and ${ }^{*} p=0.05$ of $h$ MSC-Scx versus Mock. Six to nine randomly taken $20 \times$ magnification images per section of three to five animals per group were analyzed and a total number of approximately 2000 nuclei were measured for NAR and density, while approximately 200 nuclei per group were evaluated for angular deviation.

A: Adipose areas; C: Calcaneus bone; EC: Ectopic calcification; F: Poor fibrous tissue; M: Muscle; T: Organized collagen fibers. 
single human cells incorporated within the proper of the Achilles tendons in hMSC-Mock and hMSX-Scx groups (Figure 7B).

\section{Discussion}

Due to the global population aging and weak regenerative potential of tendons, age-related degeneration, various tenopathies, traumatic and sport ruptures, tendon conditions still present major challenges to orthopedic medicine. A novel approach to treat tendon injuries is with tissue engineering, in which cells, alone or in combination with a scaffold, are introduced into the defect in order to enhance the repair and restore the original tissue function faster. In this approach a critical step is to identify the most appropriate cell type.

At present, the preferable cell types for musculoskeletal tissue engineering are adult MSC derived from bone marrow (BM) or adipose tissue (AD) [27-29], as in
(A)

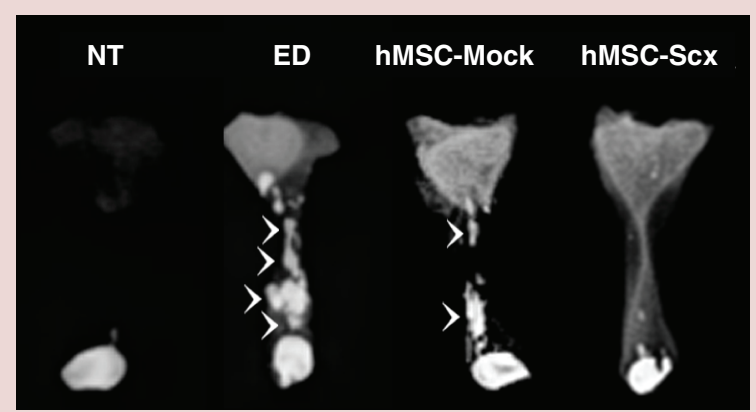

(B)

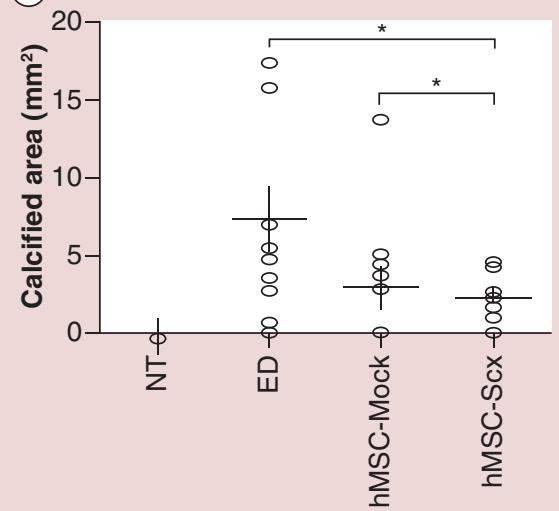

(C)

(D) CD31

(E)

CD68

占
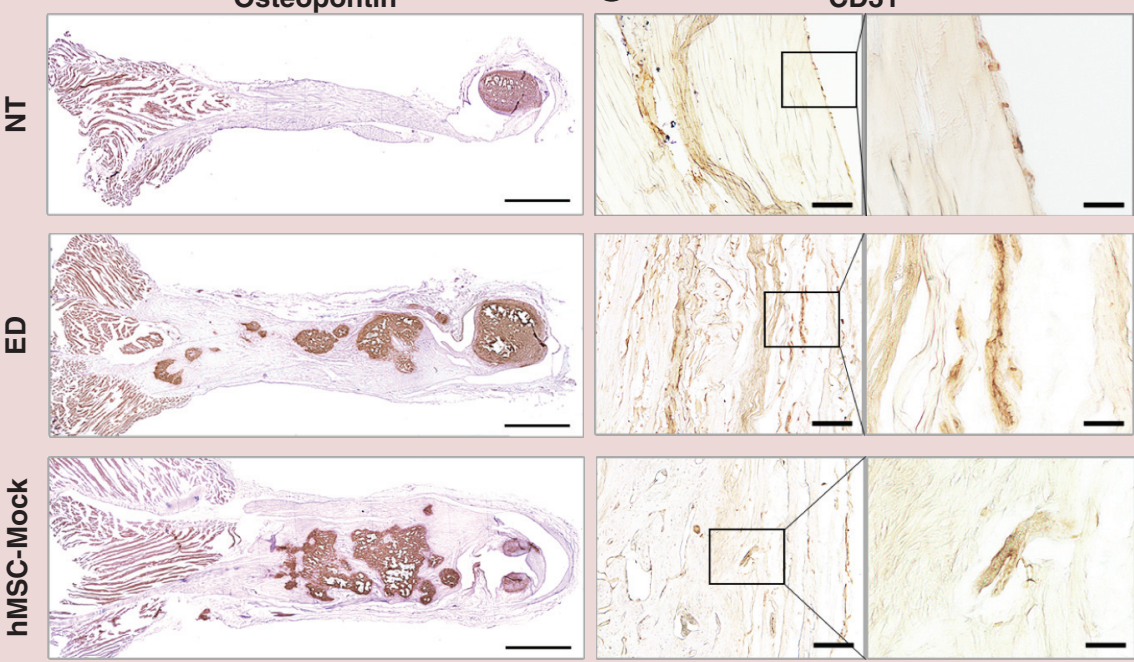

CD68
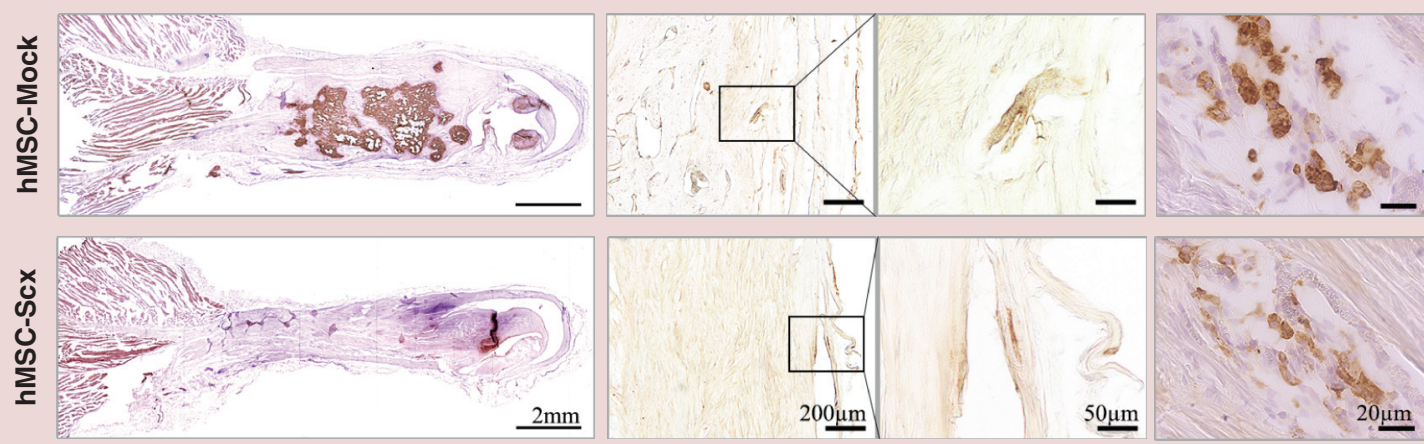

Figure 3. Evaluation ectopic calcification and vasculature within the Achilles tendons of the four study groups. (A) Representative $x$-ray images (calcification indicated with arrow heads) and (B) plot of total calcified area in each Achilles tendon measured from the $x$-ray images. Mean values \pm standard deviations (10 animals/group) are included as lines in the plot; ${ }^{*} \mathrm{p}=0.05 \mathrm{hMSC}-\mathrm{Scx}$ versus ED and hMSC-Scx versus Mock. Representative images of the bone-osteopontin (C), the vessel/endothelial CD31 (left panels are 10x magnification, right panels are 40x magnification) (D) and the macrophage CD68 markers (E). Positively stained areas are in brownish color. Stainings were reproduced at least thrice independently and for each antigen sections from three to five animals/group were analyzed. 


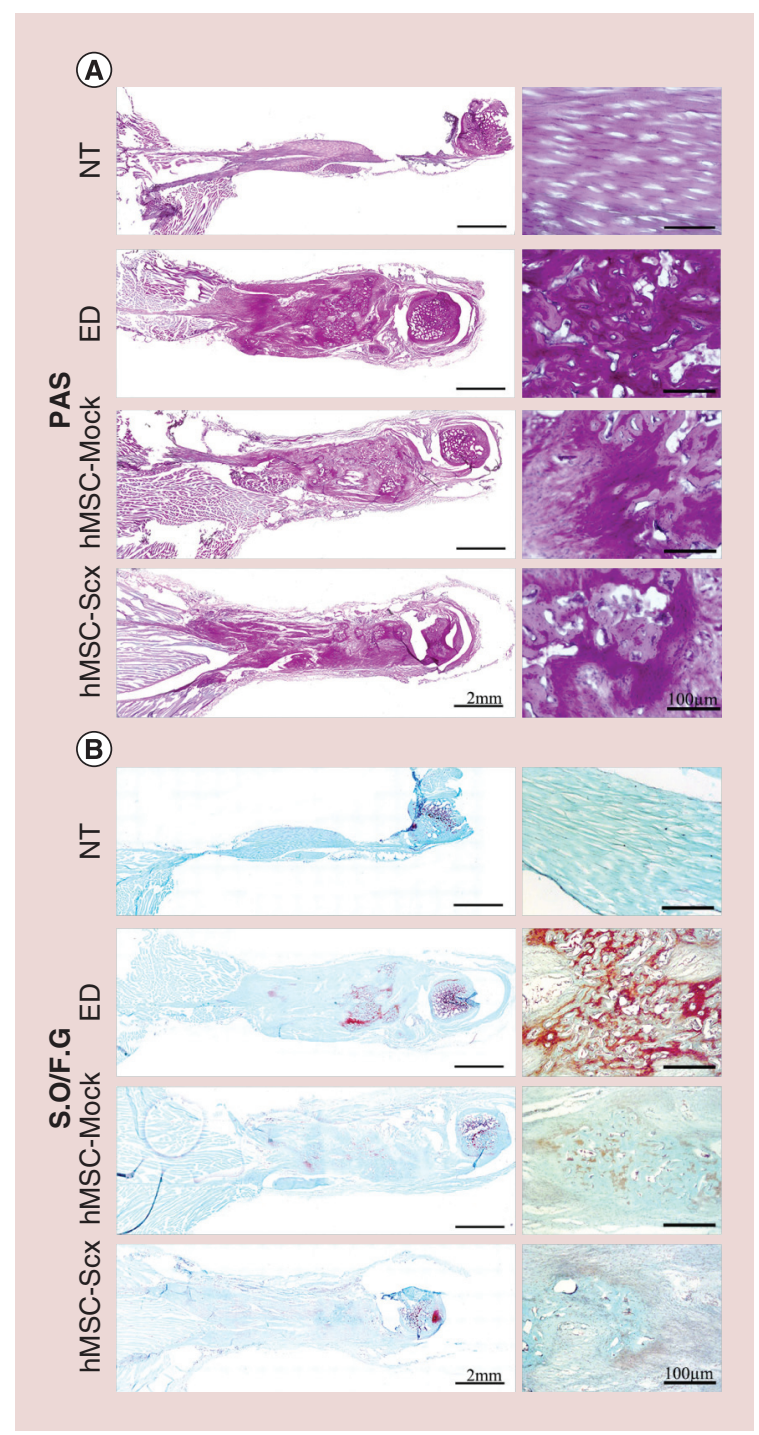

Figure 4. Analyses of mucopolysaccharides depositions and cartilage glycosaminoglycans-rich areas in the Achilles tendons of the four study groups.

Representative mosaic and magnified images of (A) Periodic Acid-Schiff (PAS, dark purple color) and (B) Safranin-O/Fast Green (S.O, orange to red color/F.G, green to light blue color) stainings. Both staining times were reproduced at least thrice independently with sections from five animals/group.

particular the BM-MSC, being the best characterized, are still the most common cell source, because they are easily accessible and expandable, and can be differentiated applying standardized protocols to at least three different mesenchymal lineages. However, there are several difficulties limiting the use of BM-MSC in tendon repair, such as ectopic ossification, bigger scar formation linked to high risk of adhesions and reduced MSC quality with donor age [30]. The results of our model demonstrated, in comparison to nonimplanted injured tendons, that delivery of MSC reduces the rate of in-tendinous ossification; however, among the two different cell types the hMSC-Scx group was superior. AD-MSC have become an alternative, very attractive cell source derived from plastic surgery, because of their low morbidity isolation procedure as well as lower risk of ectopic bone formation [30]. Studies have concluded a better collagen fiber organization and overall improvement of the tendon structure when using AD-MSC for tendon therapy [31,32]; however, this cell type also faces few challenges including endogenous tendency toward adipocyte differentiation, purity levels of the primary cells and the possible side effects on the AD-MSC quality regarding background metabolic conditions [33]. For the above reasons, in the recent years, the field has turned its attention to tendon-resident stem/progenitor cells (TSPC) [13,34], which have been suggested to play a critical role in the proliferative and remodeling phase of tendon healing [11] and have the native propensity to terminally differentiate in tendon-specific cells [11]. Indeed in our previous study, using the same full size Achilles tendon injury model, the rat tendon-derived population outperformed the classical BM-derived cells in histology and biomechanical analyzes [18]. Several hurdles are associated with the implementation of TSPC in tendon repair. On the technical/methodological side their purification and validation has proven difficult due to the lack of TSPC markers and their extended expansion has been linked to considerable phenotypic drift in vitro [35]. On the clinical side, the availability of tendon biopsies for TSPC isolation remains almost an unresolvable issue that is directly concomitant with patient comorbidity and longer preparatory procedures prior treatment, an issue that is further amplified with possible TSPC complications due to patient aging and/ or various disease conditions [13]. To overcome the difficulties associated with the use of TSPC, in 2012 we reported an alternative way to generate tendon progenitors based on the one-step programming of the classical BM-derived MSC via the ectopic expression of the leading tendon transcription factor Scx [17]. It should be noted that our single cell-derived lines are a very useful standardized experimental model. For true clinical applications, it should be explored if transient Scx delivery in autologous MSC is sufficient for their stable programming into the tendon lineage. Others have shown alternative ways to induce tenogenic commitment of hMSC; for example, overexpression of the late tendon marker EGR1 (early growth response-1) or BMP2 (bone morphogenetic protein 2)/Smad8 or combination of BMP12 and BMP13 [36-38]. Our previous work showed that subsequent to the involvement of the single early tendon factor Scx, hMSC underwent several major changes, comprising of reduced self-renewal, restricted multipotential and upregulated expression 
of tendon-related genes, which collectively advocated their successful conversion into tendon progenitors [17].

Here, we can report for the first time that after in vivo implantation, our hMSC-Scx cells not only can form tendon-like structures, but can directly contribute to enhanced tendon healing in a clinically relevant Achilles tendon injury model. Based on two different techniques for in vivo tracking, namely forensic genomic PCR and antibody-based detection, we could demonstrate that the human cells are retained within the implanted area and that a portion of these cells or their progeny can survive as long as 16 weeks post-surgery. Moreover, via a combination of tissue morphometric, $\mathrm{x}$-ray, polarized light microscopic and various histological and immunohistological analyses of proteins critical in tendon repair process we clearly showed that the hMSC-Scx group had smaller tendon diameter, negligible ectopic calcification and advanced cellular organization and matrix maturation of the injured tendons, making this study group the closest to the native noninjured tendon. Hence, we concluded that hMSC-Scx implantation aids in advanced and superior remodeling stage of the tendon healing. Based on our encouraging results, we will aim in follow-up studies to additionally optimize the application of hMSC-Scx cells in terms of: developing a suitable tendon-specific scaffold for cell delivery in order to further augment the repair outcome; evaluating their potential in a big animal model followed by end point biomechanical testing in order to approximate to the tendon dimensions of patient clinical scenarios; and establishing a transient viral or nonviral approach for $S c x$-programming to surpass the safety concerns related to genetic modification of donor-derived human cells.

The positive effect of $S c x$ in tendon healing has been suggested by three other studies. Gulotta et al. applied a transient adenoviral expression of $S c x$ in MSC to treat tendon-bone insertions of detached by surgical cut rat rotator cuffs, and evaluated tendon healing in the proliferative phase of 2 and 4 weeks. Despite the limited histological evaluation and lack of cell characterization and tracking, the main result was improved biomechanical strength in the Scx group [19]. Next, Tan et al. utilized, similar to our study, lentiviral delivery of $S c x$, but together with green fluorescent protein (GFP), in rat tendon-derived cells, in combination with fibrin glue and compared their healing potential to Mock (with GFP) and nontransduced cells in a patellar tendon window injury model with terminal analyses at 2 , 4 and 8 weeks. The authors concluded that the doubly transgenic $S c x /$ GFP tendon cells in comparison to Mock/GFP cells promoted tendon healing at the early stage. However, the data also suggested that the extent of repair after $S c x /$ GFP cell implantation is much com- parable to that of the nontransduced cells. Hence, it remains questionable if overexpression of Scx in native tendon-derived cells is beneficial and, as discussed above, a difficulty in the implication of such cells is the associated complications with their obtainment from patients' prior therapy. In addition, it should be considered that in window defect models the neighboring tendon regions stay intact, thus it is difficult to segregate the impact of local stem/progenitor cells to that of the implanted cells [21]. Last, Chen et al. tested the use of human embryonic stem cells (hESC), which were first differentiated to MSC, then engineered via lentivirus to express $S c x$ and loaded onto silk-collagen sponge for repair of a gap wound model in rat Achilles tendons at 2 and 4 weeks [20]. The in vitro pheno-

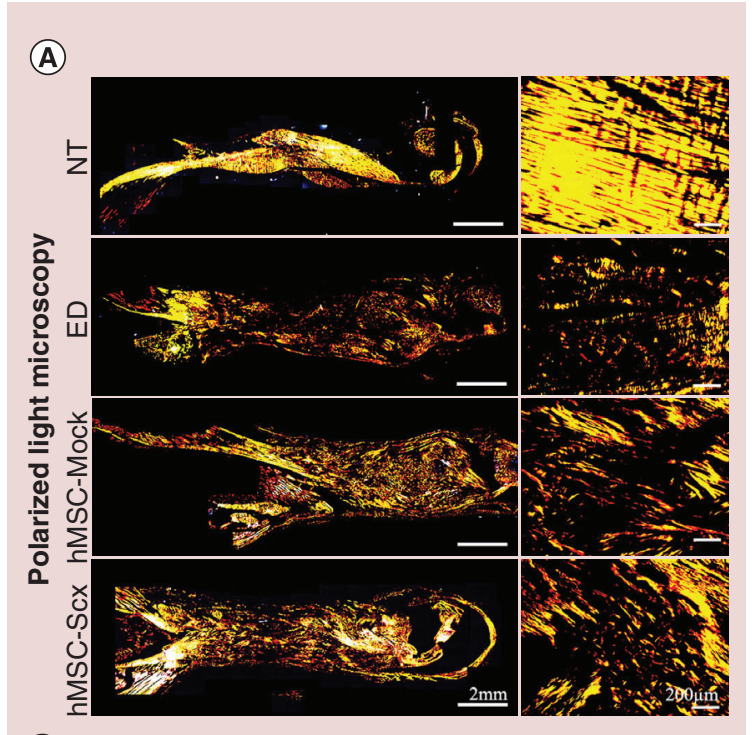

(B)

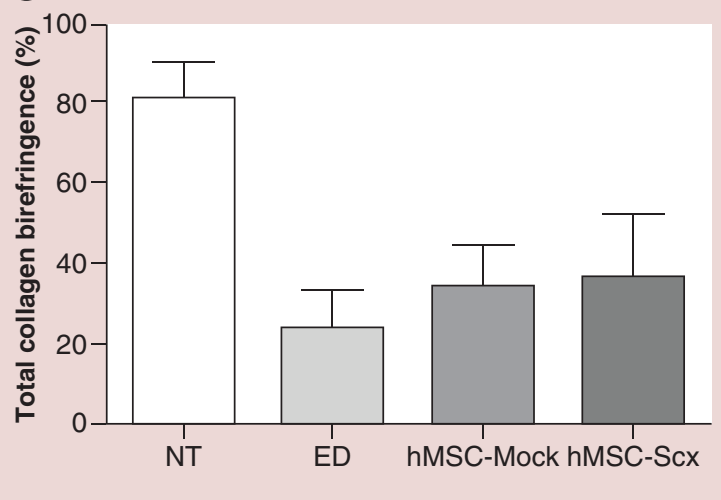

Figure 5. Microscopic evaluation and quantification of mature collagen fiber areas in the Achilles tendons. (A) Representative mosaic and magnified images of $\mathrm{H} \& \mathrm{E}-$ stained Achilles tendons under polarized light, wherein highly organized collagen fibers are visible in orange to yellow colors. (B) Quantification of total collagen birefringence (in percentage of total tendon area). Chart shows means \pm standard deviations of four animals/group. 


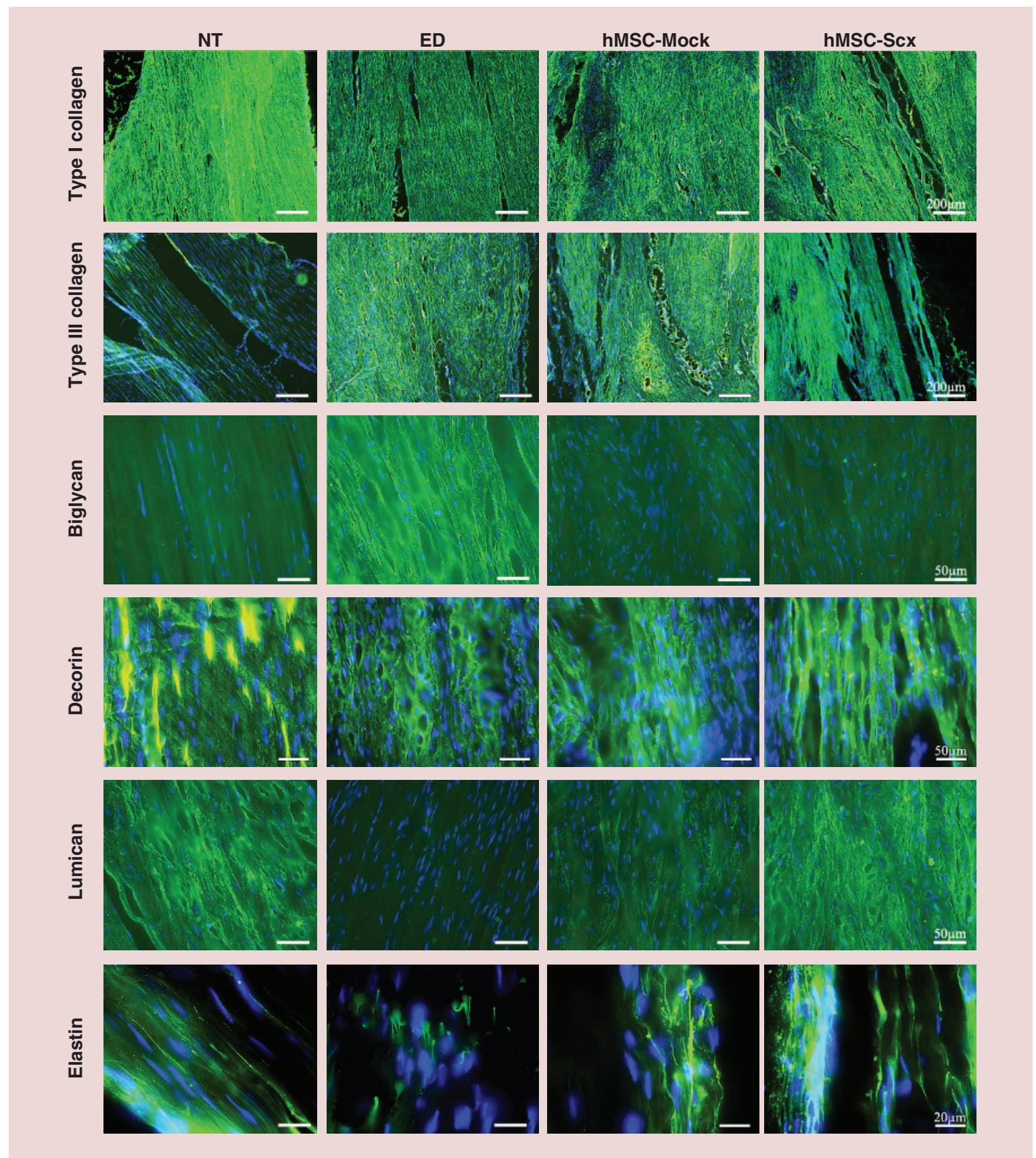

Figure 6. Immunostainings of the tendon-related matrix proteins: collagen type I and III, biglycan, decorin, lumican and elastin (all in green color) and nuclear counterstain DAPI (in blue). All stainings were reproduced at least thrice independently including sections from three to five animals/group.

typization of the hESC-MSC-Scx cells is in line with our first study [17]. At the two evaluated time points of early tendon healing, the Scx group was superior in terms of tissue organization and elastic modulus to the control GFP-transduced hESC-MSC group; it is unclear how approximating are the engineered tendons to noninjured since such were not included for direct comparison. Furthermore, since tendon repair is a time consuming process, the evaluation of the long- term effects of the cell therapy on tendon composition and strength, functional gain and repeated performance or possible injury reoccurrence has to become a key goal in future studies. In sum, our investigation provides novel and promising findings that a scaffoldfree delivery of hMSC-Scx cells for the treatment of a clinically relevant Achilles tendon injury model results in advanced late remodeling stage and overall enhancement of the tendon repair. Hence, we and others can 


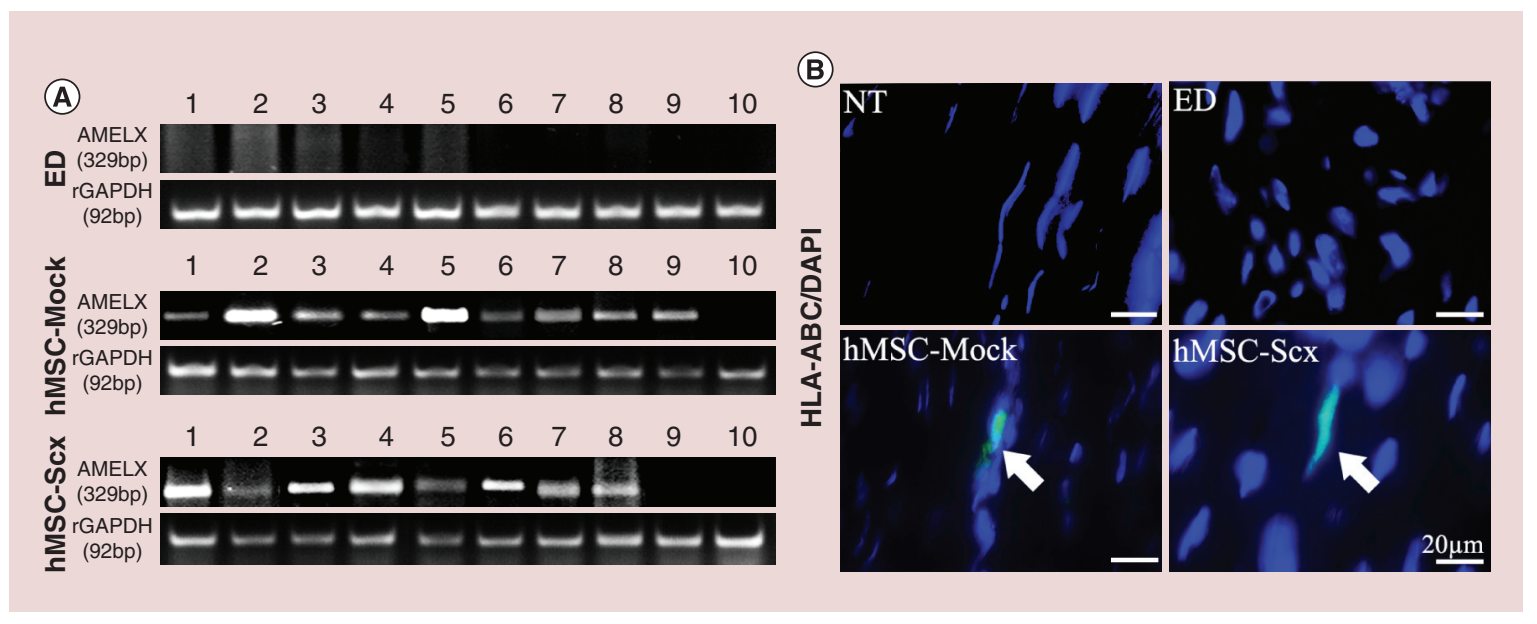

Figure 7. Detection of the implanted human cells in the rat Achilles tendons after 16 weeks in vivo. (A) By PCR for the human-specific amelogenin X (AMELX) gene using genomic DNA isolated from tendon tissue sections. Size of PCR product: $329 \mathrm{bp}$, and for simplicity not the actual animal number, but numbers 1-10 are given in the figure. For loading control PCR for rGAPDH was carried out. Size of PCR product: $92 \mathrm{bp}$. (B) By immunostaining for the human-specific antigen (HLA)-ABC. In the representative images positive human cells are in green color and the nuclear counterstain DAPI in blue color. PCR and stainings were reproduced twice with samples from 10 and five animals, respectively.

strongly propose $S c x$-delivery as a therapeutic strategy to augment tendon healing.

\section{Conclusion \& future perspective}

Scaffold-free hMSC-Scx cells implantation in injured Achilles tendons resulted in smaller size, negligible intendinous ossification and advanced tissue maturation of the newly formed tendon, which collectively suggest that hMSC-Scx cells exert strong tendon-inducting and differentiating properties making them very well suited for reconstruction of full-size tendon defects. In future, in order for hMSC-Scx cells, or variants of this technology, to become a factual clinical tool in orthopedics several open questions have to be addressed, such as possible combination and optimization with biomaterials/carriers; understanding the exact fate and survival rate of implanted cells; examining stable programming via transient $S c x$ expression in autologous cells; long-term functional and biomechanical performance tests in big animal models without immunosuppression; safety inspections behind the cell manipulation; and precise estimation of the time frame from patient hospitalization, preparation of the donors cells to exact point and technique of cell implantation. We believe that the present and forthcoming results on the application of $S c x$-based cell therapy can lead to improve the outcome of tendon surgeries.

\section{Executive summary}

- We explored the in vivo repair potential of tendon progenitors generated by a direct programming of mesenchymal stem cells with the transcription factor Scleraxis (hMSC-Scx cells).

- We utilized our clinically relevant Achilles tendon injury model and implanted scaffold-free hMSC-Scx or control hMSC-Mock cells in the injured tendons; the tendon healing was compared with empty defect (ED) and noninjured, native tendons (NT) at 16 weeks post-surgery, a time point corresponding to the late remodeling phase of the repair process.

- Via combination of morphometric, x-ray, polarized light microscopic and various histological and immunohistological analyses we found that the repaired tendons of hMSC-Scx group had smaller tendon diameters, negligible ectopic calcification and advanced cellular organization and matrix maturation, hence making this study group the closest to the NT.

- Using two different methods for in vivo tracking, we also demonstrated that the human cells are retained within the implanted area and that a portion of these cells, or their progenitors, can survive 16 weeks in vivo, suggesting their direct contribution to the healing.

- In sum, we can report that scaffold-free hMSC-Scx cell implantation aids in advanced and superior remodeling stage of the tendon healing and can be foreseen, after additional optimization steps, as a novel approach for therapy of tendon injuries. 


\section{Supplementary data}

To view the supplementary data that accompany this paper please visit the journal website at: http://www.futuremedicine.com/doi/full/10.2217/NNM.16.34

\section{Financial \& competing interests disclosure}

D Docheva acknowledged the financial support of the $A O$ Research Fund of the AO Foundation (project: S-10-74D). CF Hsieh was supported by DAAD PhD stipendship program. The authors thank C Schwarz and J Kohler for experimental help. The authors have no other relevant affiliations or financial involvement with any organization or entity with a financial interest in or financial conflict with the subject matter or materials discussed in the manuscript apart from those disclosed.

No writing assistance was utilized in the production of this manuscript.

\section{References}

1 Pennisi E. Tending tender tendons. Science 295(5557), 1011 (2002).

2 Maffulli N, Khan KM, Puddu G. Overuse tendon conditions: time to change a confusing terminology. Arthroscopy 14(8), 840-843 (1998).

3 Urwin M, Symmons D, Allison T et al. Estimating the burden of musculoskeletal disorders in the community: the comparative prevalence of symptoms at different anatomical sites, and the relation to social deprivation. Ann. Rheum. Dis. 57(11), 649-655 (1998).

4 Chard MD, Hazleman R, Hazleman BL, King RH, Reiss BB. Shoulder disorders in the elderly: a community survey. Arthritis Rheum. 34(6), 766-769 (1991).

5 Kannus P. Tendons-a source of major concern in competitive and recreational athletes. Scand. J. Med. Sci. Sports 7(2), 53-54 (1997).

6 Rees JD, Wilson AM, Wolman RL. Current concepts in the management of tendon disorders. Rheumatology (Oxford) 45(5), 508-521 (2006).

7 Docheva D, Muller SA, Majewski M, Evans CH. Biologics for tendon repair. Adv. Drug Deliv. Rev. 84, 222-239 (2015).

8 Voleti PB, Buckley MR, Soslowsky LJ. Tendon healing: repair and regeneration. Annu. Rev. Biomed. Eng. 14, 47-71 (2012).

9 Evans $\mathrm{CH}$. Cytokines and the role they play in the healing of ligaments and tendons. Sports Med. 28(2), 71-76 (1999).

10 Fenwick SA, Hazleman BL, Riley GP. The vasculature and its role in the damaged and healing tendon. Arthritis Res. $4(4), 252-260$ (2002).

11 Kajikawa Y, Morihara T, Watanabe $\mathrm{N}$ et al. GFP chimeric models exhibited a biphasic pattern of mesenchymal cell invasion in tendon healing. J. Cell. Physiol. 210(3), 684-691 (2007).

12 Sharma P, Maffulli N. Basic biology of tendon injury and healing. Surgeon 3(5), 309-316 (2005).

\section{Ethical conduct of research}

The authors state that they have obtained appropriate institutional review board approval or have followed the principles outlined in the Declaration of Helsinki for all human or animal experimental investigations (approved animal grant no. 55.21-54-2531-58-08 by the Animal Care and Use Committee of the Bavarian Government).

\section{Open access}

This article is distributed under the terms of the Creative Commons Attribution License 4.0 which permits any use, distribution, and reproduction in any medium, provided the original author(s) and the source are credited. To view a copy of the license, visit http://creativecommons.org/licenses/ by/4.0/

13 Kohler J, Popov C, Klotz B et al. Uncovering the cellular and molecular changes in tendon stem/progenitor cells attributed to tendon aging and degeneration. Aging Cell 12(6), 988-999 (2013).

14 Yao L, Bestwick CS, Bestwick LA, Maffulli N, Aspden RM. Phenotypic drift in human tenocyte culture. Tissue Eng. 12(7), 1843-1849 (2006).

15 Brent AE, Schweitzer R, Tabin CJ. A somitic compartment of tendon progenitors. Cell 113(2), 235-248 (2003).

16 Murchison ND, Price BA, Conner DA et al. Regulation of tendon differentiation by scleraxis distinguishes forcetransmitting tendons from muscle-anchoring tendons. Development 134(14), 2697-2708 (2007).

17 Alberton P, Popov C, Pragert M et al. Conversion of human bone marrow-derived mesenchymal stem cells into tendon progenitor cells by ectopic expression of scleraxis. Stem Cells Dev. 21(6), 846-858 (2012).

18 Pietschmann MF, Frankewycz B, Schmitz P et al. Comparison of tenocytes and mesenchymal stem cells seeded on biodegradable scaffolds in a full-size tendon defect model. J. Mater. Sci. Mater. Med. 24(1), 211-220 (2013).

19 Gulotta LV, Kovacevic D, Packer JD, Deng XH, Rodeo SA. Bone marrow-derived mesenchymal stem cells transduced with scleraxis improve rotator cuff healing in a rat model. Am. J. Sports Med. 39(6), 1282-1289 (2011).

20 Chen X, Yin Z, Chen JL et al. Scleraxis-overexpressed human embryonic stem cell-derived mesenchymal stem cells for tendon tissue engineering with knitted silkcollagen scaffold. Tissue Eng. A 20(11-12), 1583-1592 (2014).

21 Tan C, Lui PP, Lee YW, Wong YM. SCX-transduced tendonderived stem cells (TDSCS) promoted better tendon repair compared with mock-transduced cells in a rat patellar tendon window injury model. PLoS ONE 9(5), e97453 (2014).

22 Ozeki N, Muneta T, Koga H et al. Transplantation of Achilles tendon treated with bone morphogenetic protein 7 promotes meniscus regeneration in a rat model of massive meniscal defect. Arthritis Rheum. 65(11), 2876-2886 (2013). 
Stoll C, John T, Conrad C et al. Healing parameters in a rabbit partial tendon defect following tenocyte/biomaterial implantation. Biomaterials 32(21), 4806-4815 (2011).

24 Haas-Rochholz H, Weiler G. Additional primer sets for an amelogenin gene PCR-based DNA-sex test. Int. J. Legal Med. 110 (6), 312-315 (1997).

25 Yoon JH, Halper J. Tendon proteoglycans: biochemistry and function. J. Musculoskelet. Neuronal Interact. 5(1), 22-34 (2005).

26 Juneja SC, Veillette C. Defects in tendon, ligament, and enthesis in response to genetic alterations in key proteoglycans and glycoproteins: a review. Arthritis 2013, 154812 (2013).

27 Yang G, Rothrauff BB, Lin H, Gottardi R, Alexander PG, Tuan RS. Enhancement of tenogenic differentiation of human adipose stem cells by tendon-derived extracellular matrix. Biomaterials 34(37), 9295-9306 (2013).

28 Chaudhury S. Mesenchymal stem cell applications to tendon healing. Muscles Ligaments Tendons J. 2(3), 222-229 (2012).

29 Mazzocca AD, Mccarthy MB, Chowaniec D et al. Bone marrow-derived mesenchymal stem cells obtained during arthroscopic rotator cuff repair surgery show potential for tendon cell differentiation after treatment with insulin. Arthroscopy 27(11), 1459-1471 (2011).

Harris MT, Butler DL, Boivin GP, Florer JB, Schantz EJ, Wenstrup RJ. Mesenchymal stem cells used for rabbit tendon repair can form ectopic bone and express alkaline phosphatase activity in constructs. J. Orthop. Res. 22(5), 998-1003 (2004).
31 Nixon AJ, Dahlgren LA, Haupt JL, Yeager AE, Ward DL. Effect of adipose-derived nucleated cell fractions on tendon repair in horses with collagenase-induced tendinitis. Am. J. Vet. Res. 69(7), 928-937 (2008).

32 Behfar M, Sarrafzadeh-Rezaei F, Hobbenaghi R, Delirezh N, Dalir-Naghadeh B. Enhanced mechanical properties of rabbit flexor tendons in response to intratendinous injection of adipose derived stromal vascular fraction. Curr. Stem Cell Res. Ther. 7(3), 173-178 (2012).

33 Uysal AC, Mizuno $H$. Tendon regeneration and repair with adipose derived stem cells. Curr. Stem Cell Res. Ther. 5(2), 161-167 (2010)

34 Bi Y, Ehirchiou D, Kilts TM et al. Identification of tendon stem/progenitor cells and the role of the extracellular matrix in their niche. Nat. Med. 13(10), 1219-1227 (2007).

35 Tan Q, Lui PP, Rui YF. Effect of in vitro passaging on the stem cell-related properties of tendon-derived stem cells implications in tissue engineering. Stem Cells Dev. 21(5), 790-800 (2012).

36 Guerquin MJ, Charvet B, Nourissat G et al. Transcription factor EGR1 directs tendon differentiation and promotes tendon repair. J. Clin. Invest. 123(8), 3564-3576 (2013).

37 Hoffmann A, Pelled G, Turgeman G et al. Neotendon formation induced by manipulation of the $S \operatorname{mad} 8$ signalling pathway in mesenchymal stem cells. J. Clin. Invest. 116(4), 940-952 (2006).

38 Haddad-Weber M, Prager P, Kunz M et al. BMP12 and BMP13 gene transfer induce ligamentogenic differentiation in mesenchymal progenitor and anterior cruciate ligament cells. Cytotherapy 12(4), 505-513 (2010). 